\title{
Imaging the Infant Retina with a Hand-held Spectral-Domain Optical Coherence Tomography Device
}

\author{
ADRIENNE W. SCOTT, SINA FARSIU, LAURA B. ENYEDI, DAVID K. WALLACE, AND CYNTHIA A. TOTH
}

- PURPOSE: To evaluate and treat infant retina through the use of a hand-held spectral-domain optical coherence tomography (SD OCT) device in selected cases of Shaken Baby syndrome (SBS).

- DESIGN: Observational case series.

- METHODS: A novel SD OCT system was optimized and evaluated for infant imaging. An adult eye was imaged with both a standard clinical SD OCT system and the hand-held system. Four eyes of two infants with a history of SBS were imaged with the hand-held system. One infant was imaged again during follow-up examinations. Robust image processing algorithms were developed to create high-quality images. Images were assessed for usefulness in demonstrating pertinent morphologic features.

- RESULTS: The novel SD OCT unit proved effective for data acquisition and comparable with conventional chinrest SD OCT. Rapid data acquisition limited motion artifact within the B-scan, although there was slight motion between B scans. The SD OCT images provided previously unseen details with regard to the morphologic features of retinal lesions in these infant eyes. This information influenced prognosis and management.

- CONCLUSIONS: As with adults, the hand-held customized SD OCT proved to be an invaluable tool in the differentiation of disease processes or injury in these eyes under study. SD OCT imaging systems may be considered a useful adjunct to RetCam fundus photography for assessment and clinical management in cases of SBS. (Am J Ophthalmol 2009;147:364-373. (C) 2009 by Elsevier Inc. All rights reserved.)

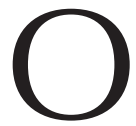

PTICAL COHERENCE TOMOGRAPHY (OCT) IS A diagnostic imaging method that provides information about the vitreoretinal interface and the architecture of the retina and retinal pigment epithelium (RPE) beyond what can be characterized fully by biomicroscopy alone. ${ }^{1}$ OCT provides in vivo information about retinal morphologic features in a rapid, noninvasive, and noncontact manner. Thus, it is an ideal tool for charac-

Accepted for publication Aug 5, 2008.

From the Department of Ophthalmology, Albert Eye Research Institute, Duke University Eye Center, Duke University Medical Center (A.W.S., S.F., L.B.E., D.K.W., C.A.T.); and the Department of Biomedical Engineering, Pratt School of Engineering, Duke University (C.A.T.), Durham, North Carolina.

Inquiries to Cynthia A. Toth, Duke University Eye Center, Box 3802, Durham, NC 27710; e-mail: cynthia.toth@duke.edu terization of retinal pathologic features in children. Shields and associates performed time-domain (TD) OCT on a series of 56 eyes of 56 children younger than 18 years (range, four to seven years). ${ }^{2}$ They found that OCT was well-tolerated in children and was more sensitive than clinical examination in detection of macular pathologic features such as posterior vitreous detachment, surface wrinkling maculopathy, cystoid and noncystoid macular edema, subfoveal fluid, and retinal thinning. TD OCT also has been performed in anesthetized infants with advanced retinopathy of prematurity. It has been useful in demonstrating posterior hyaloid contracture ${ }^{3}$ and macular anatomic abnormalities that may influence surgical planning and may predict visual outcomes after lens-sparing vitrectomy. ${ }^{4,5}$ In 2002, we attempted to use a conventional TD OCT suspended on a surgical arm while examining infants under anesthesia, but we failed to achieve the stable platform necessary for reproducible examinations.

Recently, spectral-domain (SD) OCT has provided higher-resolution images (less than $5-\mu \mathrm{m}$ axial resolution) with rapid image acquisition useful for both 2-dimensional (2D) and 3-dimensional (3D) imaging. Because data acquisition is more than 40 times faster with SD OCT than with TD OCT, one can image across larger areas of the retina or scan smaller areas at much greater density. ${ }^{6-9}$ SD OCT has been used to document spontaneous closure of idiopathic macular holes in adult patients and also has been used to document an optic nerve pit with a macular schisis-like cavity in an adult patient. ${ }^{8,9} \mathrm{We}$ hypothesized that a portable, hand-held SD OCT system accompanied by novel image processing algorithms could be used to image and evaluate effectively the extent of retinal pathologic features in infants with Shaken Baby syndrome (SBS). We also investigated whether the system would be stable enough for lesion localization.

\section{METHODS}

AFTER INFORMED CONSENT WAS OBTAINED, THE STUDY was performed in one adult and two pediatric subjects. The adult subject was imaged while sitting upright using conventional TD OCT (Zeiss Meditec Inc, Dublin, California, USA) and table-based clinical SD OCT (Bioptigen Inc, Research Triangle Park, North Carolina, USA), and while lying supine using a research hand-held, noncontact SD OCT system (Bioptigen Inc). Note that 


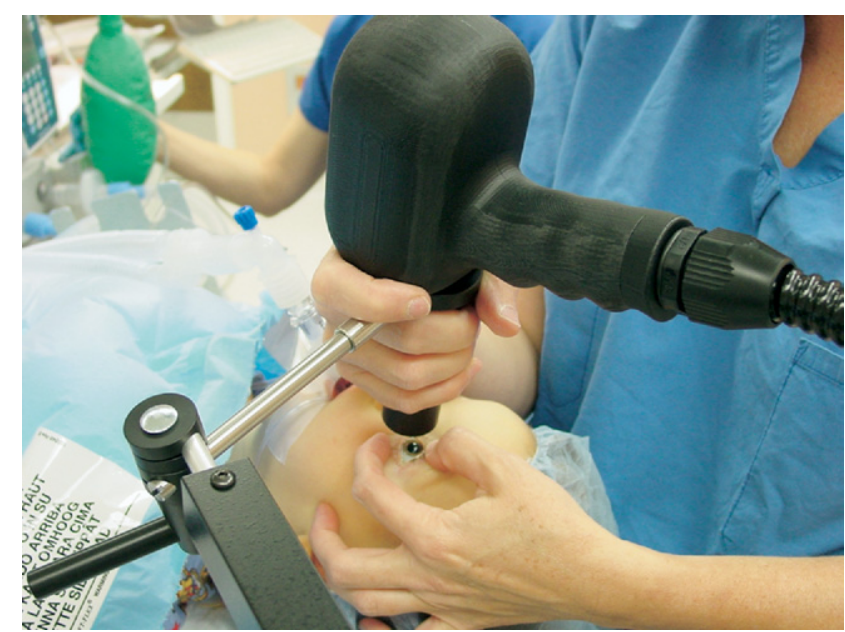

FIGURE 1. Photograph showing the hand-held spectral-domain optical coherence tomography (SD OCT) being used to image an infant eye. The infant is supine and under endotracheal anesthesia. The imaging hand piece is held over the infant's eye while the SD OCT output is observed on a computer screen on an adjacent wheeled cart.

the term hand-held refers to the SD OCT system that contains both a moveable imaging hand piece that is connected via a 1.3 -m flexible fiberoptic cable to a moveable cart holding the SD OCT system and viewing screen. The hand-held OCT scanning head operates while positioned at any angle and thus is useful for scanning in the supine patient. The pediatric subjects were supine while imaged with the hand-held SD OCT system (Figure 1). While imaging, the operator could use either an armature to stabilize the hand, as shown in Figure 1, or simply could stabilize the hand by resting several fingers on the subject's forehead. The latter is preferred by this group because of the ease of movement to adjust the scan angle. The SD OCT system operated at the same specifications when used for table-based imaging or handheld imaging. For pediatric SD OCT imaging, the system was optimized by manually adjusting the focal length of the imaging hand piece for the refractive error of the eye. The final focus adjustment was made on the hand piece while observing for optimal brightness of the SD OCT scans in real-time. The reference arm was shortened to correct for the coarse estimate of decrease in axial length of the pediatric eye. The reference arm length was refined by adjusting the reference arm to minimize visible iris shadowing on the SD OCT scans in real-time while the hand piece was adjusted horizontally.

To achieve high-resolution low-noise images, we used two SD OCT imaging schemes, namely volumetric and lateral-repeated scanning techniques (Figure 2). In volumetric scanning, sequences are captured as progressively azimuthally translated B scans, creating a 3D representation of the retinal layers (Figure 2, Top). Note that by axially projecting such $3 \mathrm{D}$ volumetric scans (averaging each A-scan), a 2D image analogous to a fundus image can be created. This image, called the sum voxel projection (SVP), is especially useful for scan orientation and localization of retinal structures or pathologic features (Figure 2, Top). ${ }^{10,11}$ Moreover, the retinal vessel pattern manifested on the SVP is a good measure for evaluating the 3D imaging quality. For example, motion artifacts in a volumetric imaging experiment often will result in broken or jagged blood vessel representation on the SVP.

Although volumetric scanning provides invaluable 3D geometric information about preretinal, intraretinal, and subretinal structures, each individual B-scan is relatively very noisy. Alternative to the volumetric imaging scheme, it is possible to capture a sequence of repeated B scans from a unique azimuthal position (lateral repeated scan). In a postprocessing step, these images are registered and summed (fused), creating a unique, less noisy image (Figure 2, Middle). We determined the location of such B scans approximately with respect to the SVP of a corresponding volumetric scan by identifying a match between the lateral repeated B scans and the closest corresponding scan from within the volume.

In our experiments, we performed an SD OCT volumetric scan of a $10 \times 10-\mathrm{mm}$ retinal area of each eye in fewer than 6 seconds. This produced $100 \mathrm{~B}$ scans $(1000 \mathrm{~A}$ scans each) with oversampling along the lateral axis and $80-\mu \mathrm{m}$ spacing between B scans. Moreover, several sets of lateral repeated scans at different azimuthal positions were captured in the same field of view and lateral and axial resolutions as the volumetric scans.

For hand-held imaging, robust image processing algorithms were used to visualize better the $3 \mathrm{D}$ scans and to fuse the information from repeated scans, creating higherquality images. The quality of the SVP images was enhanced by applying two adaptive image processing algorithms. First, the raw SD OCT images were denoised using an iterative maximum a posteriori-based algorithm, exploiting L2 norm penalty and a variation of the Tikhonov prior as the likelihood and regularization terms, respectively. ${ }^{12}$ In this article, we refer to these individually denoised images as enhanced B scans. Then, the contrast of the SVP image was enhanced by comparing and normalizing the intensity of each projected B-scan (horizontal lines on the SVP) with respect to the global intensity of the SVP. In this fashion, the erroneously dark or bright outlier frames are adjusted with respect to the intensity of their neighboring projected B scans. ${ }^{13}$ To create summed images from the lateral repeated scans, large motions were corrected using the ImageJ (freeware; National Institutes of Health, Bethesda, Maryland, USA) StackReg registration plug-in. ${ }^{14}$ Then, these warped images were registered with subpixel accuracy using a previously described method ${ }^{15}$ and were fused using the second-order classic kernel regression algorithm. ${ }^{16}$ 


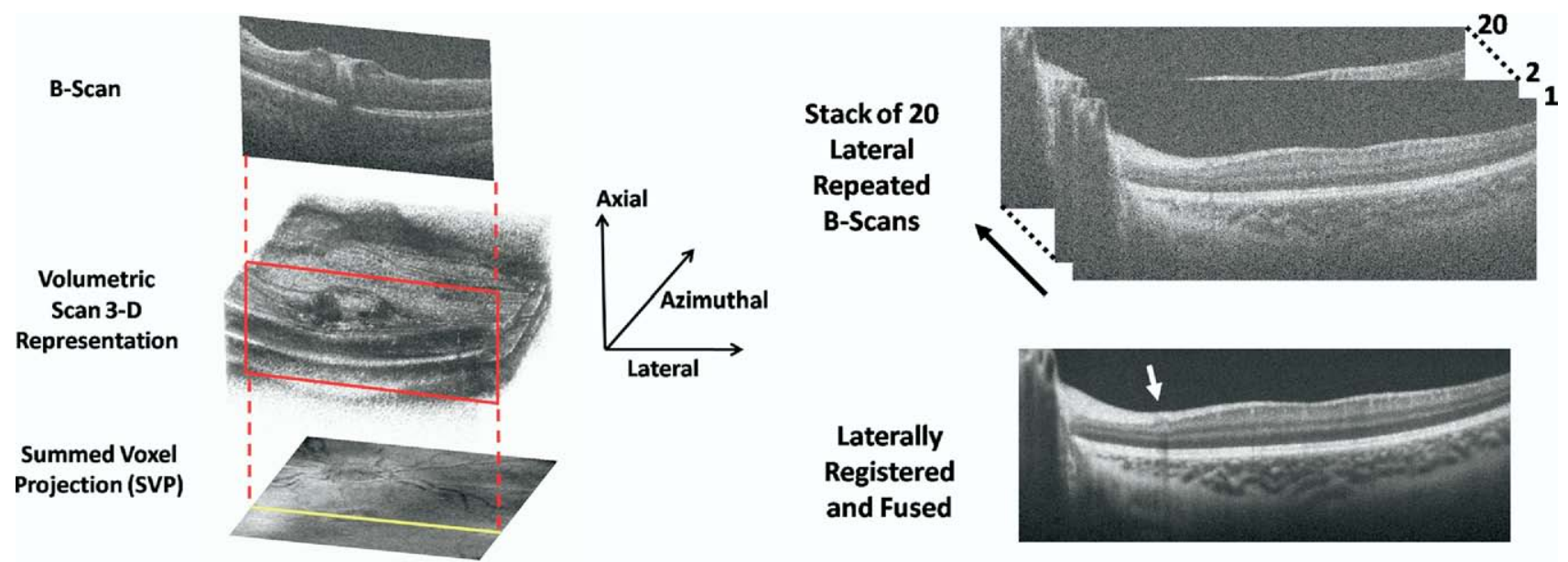

FIGURE 2. Volumetric and lateral repeated SD OCT scanning schemes. (Left) Volumetric SD OCT scanning scheme: (Top left) a sequence of azimuthally positioned B scans is captured to create (Middle left) a 3-dimensional (3D; volumetric) representation of the macula. The location of the selected B-scan is outlined in red in the volume. (Bottom left) The sum voxel projection (SVP) image was created by axially averaging the $3 \mathrm{D}$ volume. Because the B scans actually generate the SVP image, each B-scan corresponds to a single band (yellow line) on the SVP. Note that the retinal vessels cast shadows in the B scans, and this shadowing (best seen on the Right) produces the vessel pattern in the SVP. (Right) Lateral repeated SD OCT scanning scheme: (Top right) a sequence of B scans from a unique azimuthal position is captured, registered, and fused, creating (Bottom right) a less noisy image. A retinal vessel with shadowing is visible at the white arrow.

\section{RESULTS}

- ADUlT IMAGING: The pilot testing of the hand-held SD OCT system was performed in an adult subject who had a full-thickness macular hole in one eye. Imaging was performed with the examiner seated beside the patient and with the subject positioned supine on a stretcher. Images from the hand-held SD OCT system were compared with images captured with a conventional TD and tabletop SD OCT (Figure 3). The hand-held SD OCT system was portable on a wheeled cart. The hand-held probe was aligned over the eye with relative ease. Images were acquired quickly and were of excellent quality, although there was greater anteroposterior shift in image location from frame to frame with the hand-held system when compared with the chin-rest tabletop system. Images of the full-thickness macular hole using the hand-held SD OCT were similar in quality as compared with images obtained with the table-based SD OCT system. SD OCT images of the retina clearly were of improved quality compared with traditional TD OCT of this eye (Figure 3).

- PEDiatric IMAging: Case 1. A 14-month-old female infant was evaluated for ocular sequelae of SBS. Eight months before this examination, in an emergency room visit, the infant was found to have subdural hemorrhage on magnetic resonance scan, left radial and right humeral fractures on skeletal survey, and bilateral vitreous and subhyaloid hemorrhages. The vitreous and subhyaloid hemorrhages had resolved in both eyes over the preceding eight months; however, the child had continued decreased vision in her right eye. She demonstrated a preference for the left eye, and visual acuity (VA) by Teller acuity measured 0.31 cycles per degree in the right eye and 6.4 cycles per degree in the left eye. A macular lesion in the right eye was noted in the clinic and was evaluated during an examination under anesthesia (EUA). Examination with indirect ophthalmoscopy of the right eye demonstrated perimacular folds in a defined ring around the macula and a hyperpigmented foveal lesion that appeared to be at the level of the RPE (Figure 4, Top left). The differential diagnosis for this lesion included full-thickness macular hole, scar from previous subfoveal hemorrhage, foveal cyst, and epiretinal membrane (ERM) with pseudohole. Fundus examination of the left eye showed a subtle increase in macular pigmentation and preretinal gliosis at an arcuate perimacular retinal fold superior and temporal to the macula (Figure 5, Top left). There was no retinal tear or retinal detachment observed in either eye.

Hand-held SD OCT images of the right eye showed a highly reflective stalk (of probable chorioretinal fibrotic tissue) centered within a full-thickness chronic macular hole (Figure 4). Hand-held SD OCT images of the left eye demonstrated a lamellar hole and partial separation of the posterior hyaloid (Figure 5). There was no evidence of vitreomacular traction, cystoid macular edema, or retinal elevation. Because of the chronic nature of the macular hole and the guarded visual prognosis, no surgical intervention was pursued.

Case 2. A 6-month-old male infant was evaluated for ocular sequelae of SBS. During an emergency room visit one month earlier, the infant was found to have bilateral 

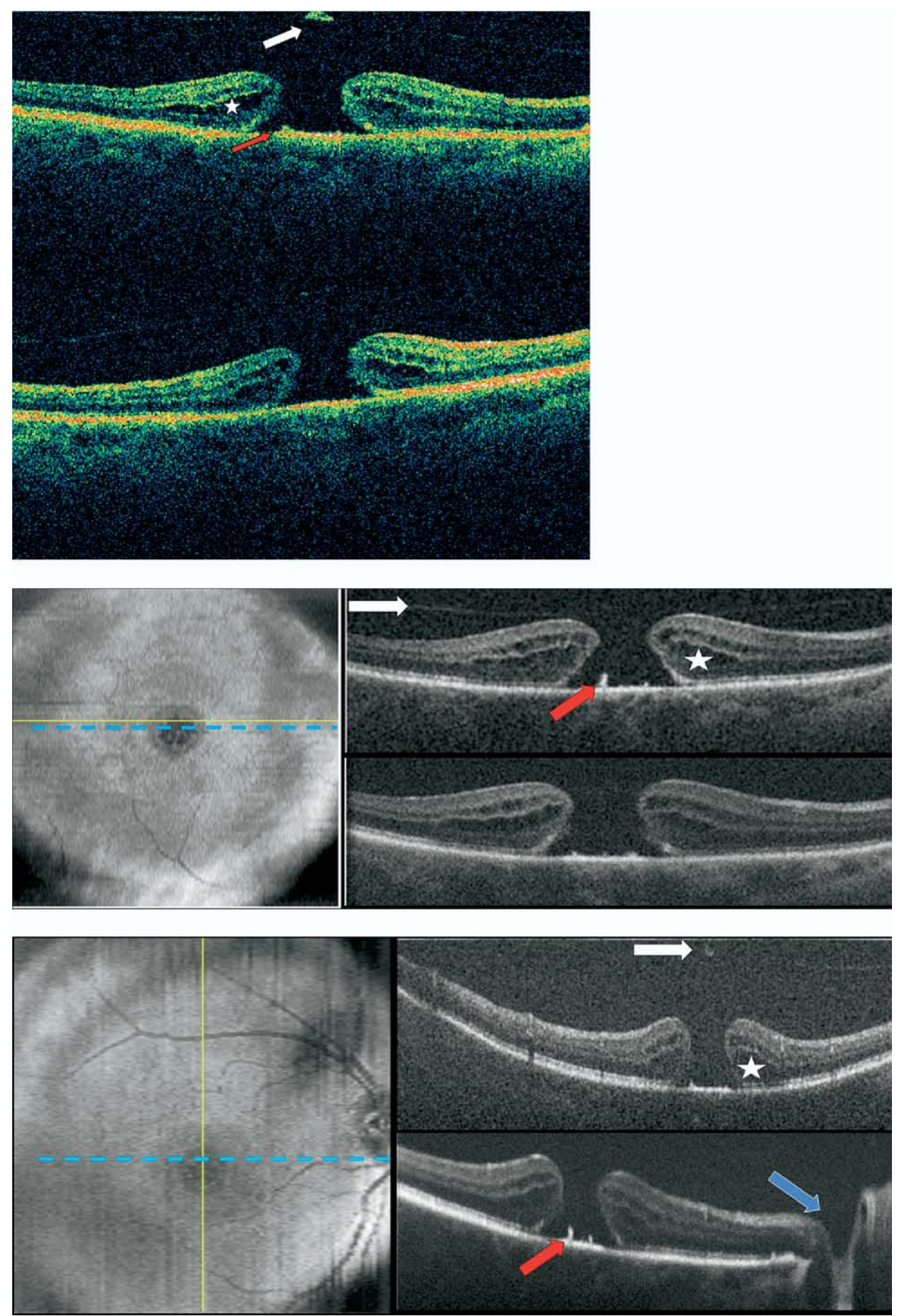

FIGURE 3. OCT scans of a full-thickness macular hole in the right eye of an adult patient. (Top) Conventional time-domain OCT: (Top) horizontal and (Top bottom) vertical Stratus OCT 3 scans. Each line scan is $6 \mathrm{~mm}$ in length and is a cross-section composed of 512 A scans. (Middle) Tabletop SD OCT obtained in the clinic. (Middle left) The SVP is of a $5 \times 5$-mm area, constructed from $200 \mathrm{~B}$ scans of $500 \mathrm{~A}$ scans each. The (Middle, top right) yellow line marks the exact location of the enhanced B-scan (sweeping temporal to nasal), and the (Middle, bottom right) blue line represents the approximate location of the lateral repeated image (sweeping temporal to nasal). (Bottom) Hand-held SD OCT image obtained from the supine subject on the operating table. (Bottom left) The SVP is of a $10 \times 10-\mathrm{mm}$ area, constructed from $100 \mathrm{~B}$ scans of $1000 \mathrm{~A}$ scans each. The (Bottom top right) yellow line marks the exact location of the enhanced B-scan (sweeping superior to inferior), whereas the (Bottom right) blue line represents the approximate location of the lateral repeated image (sweeping temporal to nasal). The blue arrow points to the optic nerve. In all three OCT image sets, cystoid macular edema is noted at the edges of the macular hole (star), small hyperreflective lesions are noted in the base of the macular hole at the level of the retinal pigment epithelium (red arrow), and hyperreflective partial posterior hyaloid separation (white arrow) with a possible operculum is evident. 


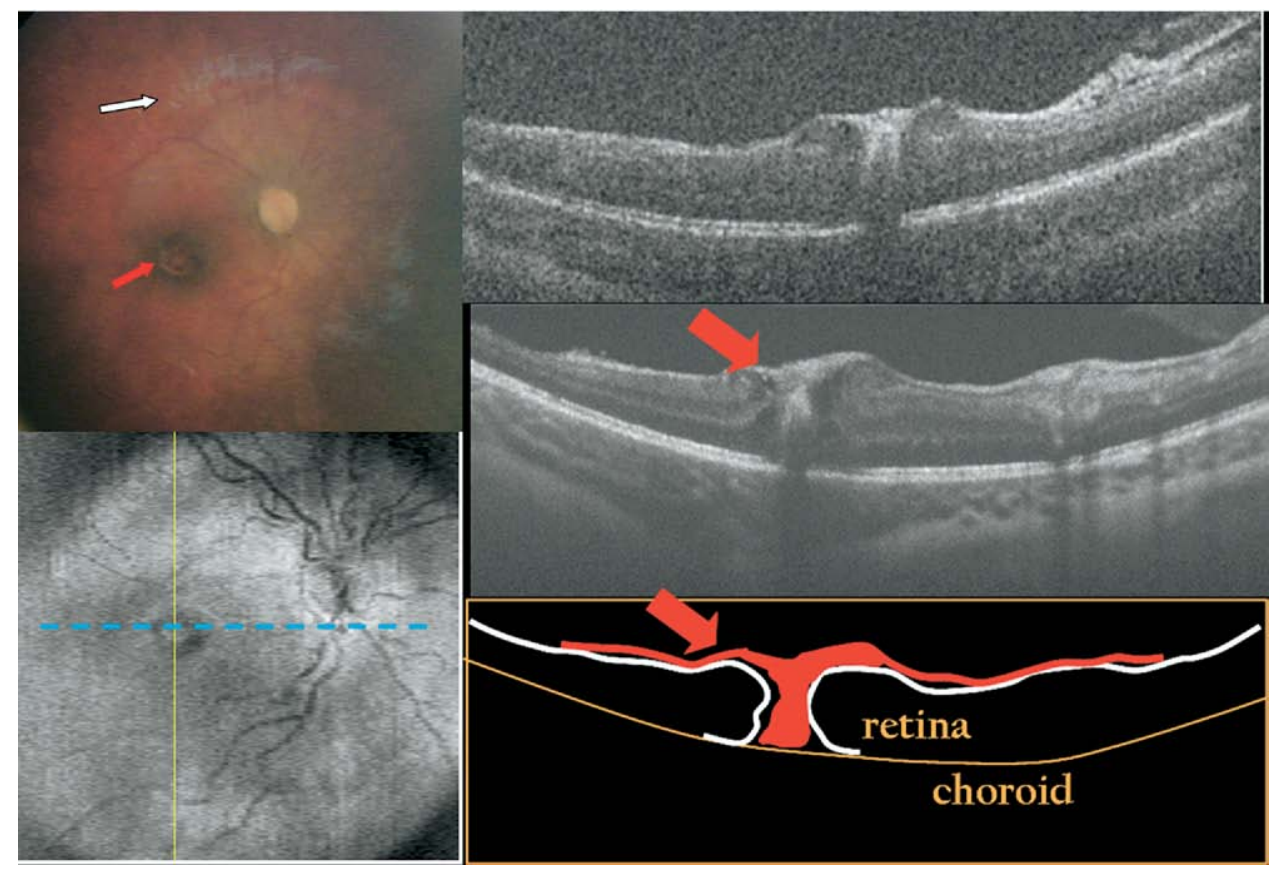

FIGURE 4. (Top left) RetCam photograph of a female infant with Shaken Baby syndrome (SBS) showing a hyperpigmented perifoveal ring (red arrow) and a white, elevated ring outside the major vascular arcades consistent with a perimacular fold. The (Bottom left) SVP, (Top right) enhanced B-scan, (Middle right) and registered-summed lateral repeated image are shown. The yellow line on the SVP marks the exact location of the enhanced B-scan (sweeping from inferior to superior), whereas the blue line represents the approximate location of the lateral repeated image (sweeping from temporal to nasal). These SD OCT images revealed a highly reflective stalk of tissue (probably chorioretinal fibrotic tissue; yellow arrow) centered within a full-thickness chronic macular hole. (Bottom right) Schematic representation.

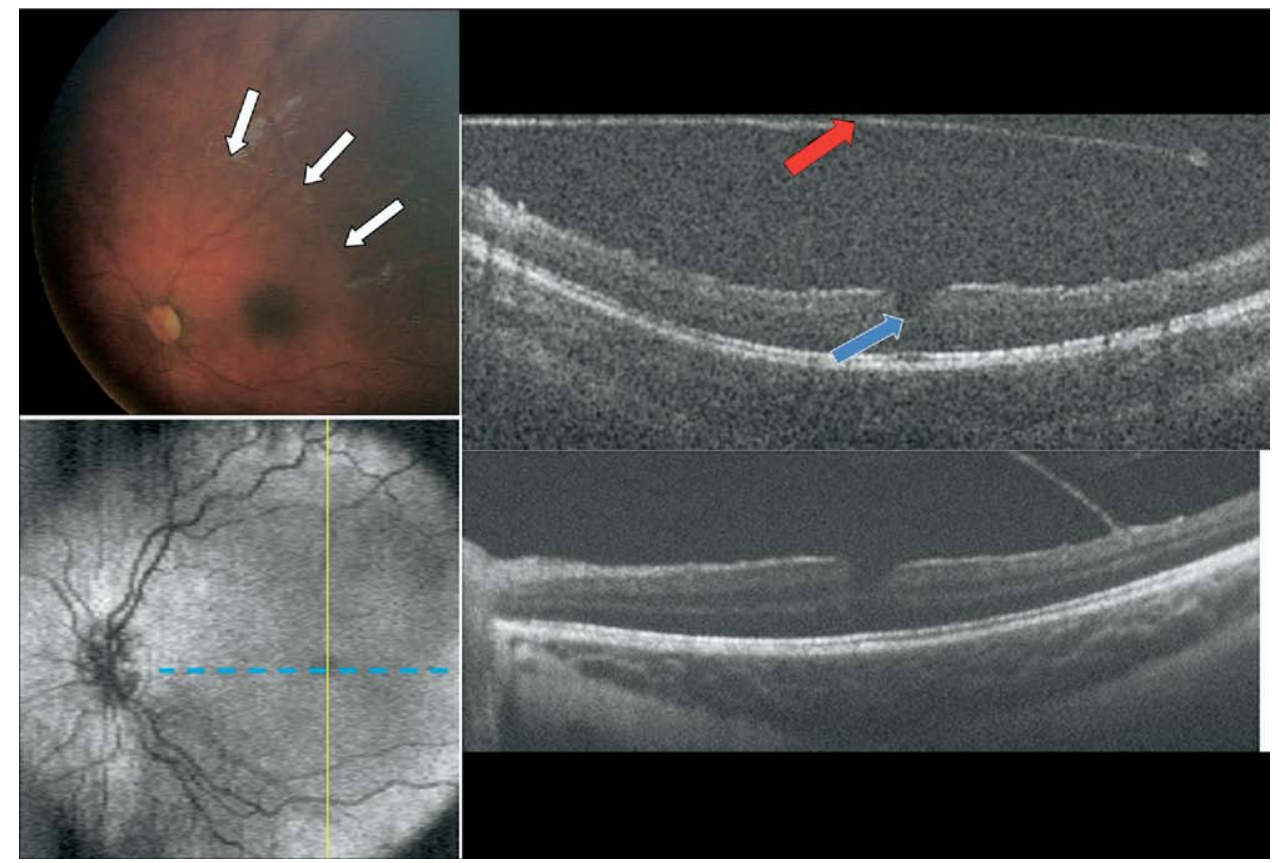

FIGURE 5. (Top left) RetCam photographs of the left eye of a female infant with SBS showing a perimacular fold (white arrows). The (Bottom left) SVP image, (Top right) enhanced B-scan, and (Bottom right) registered-summed lateral repeated image are shown. The yellow line on the SVP marks the exact location of the enhanced B-scan (sweeping from superior to inferior), whereas the blue line represents the approximate location of the lateral repeated image (sweeping nasal to temporal). The SD OCT images reveal a partial posterior hyaloid separation (red arrow) and a lamellar macular hole (blue arrow). 


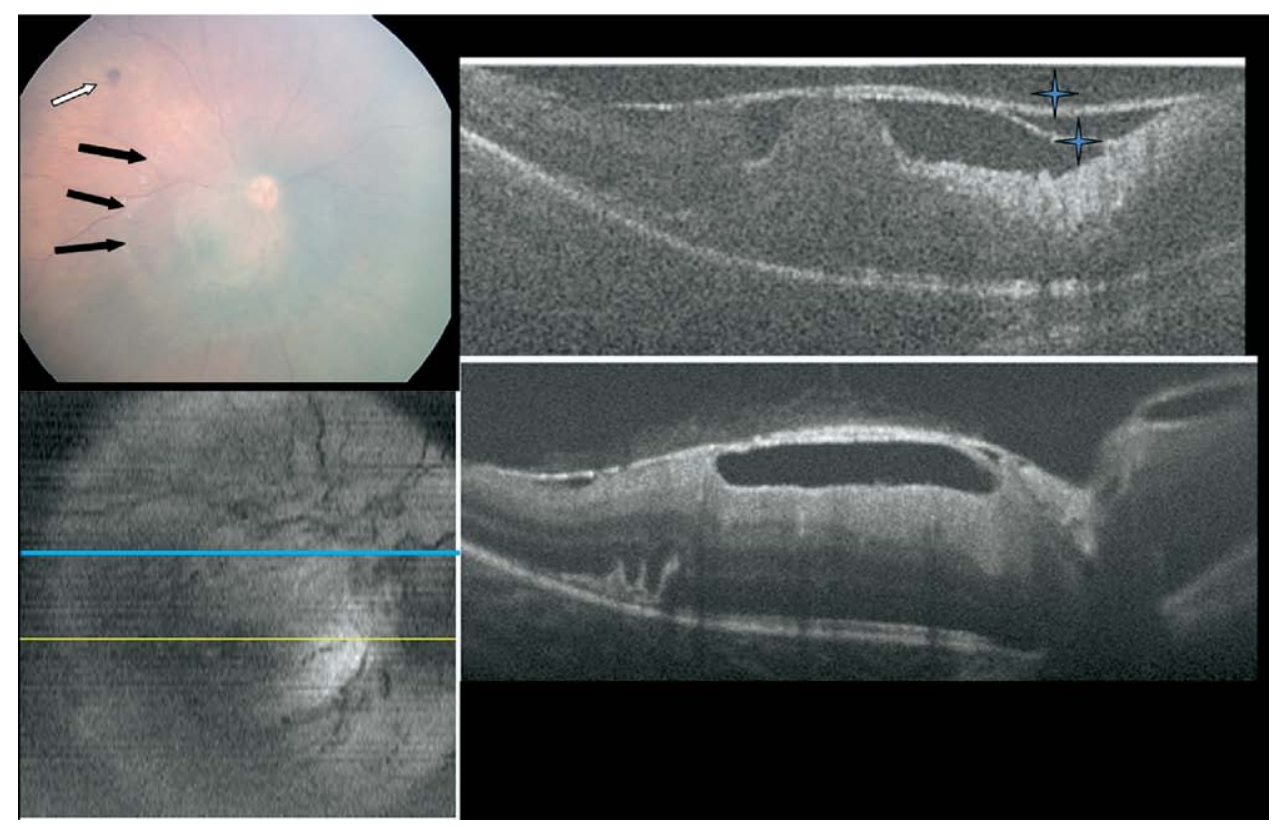

FIGURE 6. (Top left) RetCam photograph of the right eye of a male infant with SBS showing a supertemporal intraretinal hemorrhage (white arrow), a perimacular fold (black arrows), tortuous macular vessels, and an epiretinal membrane (ERM). The (Bottom left) SVP image, (Top right) enhanced B-scan, and (Bottom right) registered-summed lateral repeated image are shown. The yellow line on the SVP marks the exact location of the enhanced B-scan, whereas the blue line represents the approximate location of the lateral repeated image (both sweeping temporal to nasal). The SD OCT images reveal a thickened macula and disruption of the normal foveal architecture by a double layer of ERM (stars).

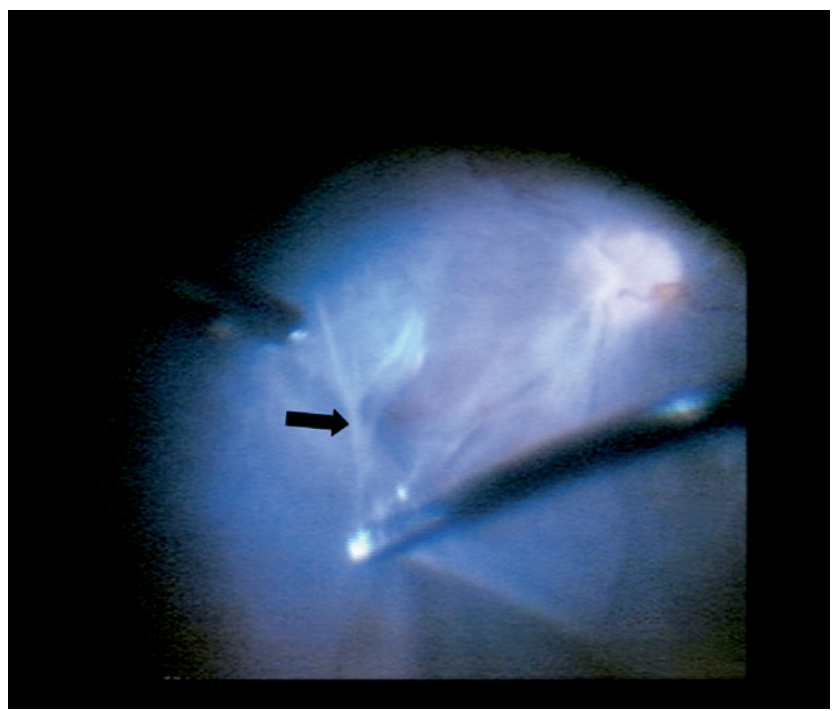

FIGURE 7. A layer of ERM (black arrows) is peeled from the retinal surface with forceps in the eye of the male infant with SBS.

subdural hematomas on both computed tomographic and magnetic resonance imaging scans of the brain, ulnar and femur fractures on skeletal survey, and bilateral intraretinal hemorrhages without vitreous hemorrhage. The infant was noted to fix and follow light in the left eye, but not with the right eye. Teller acuity card VA revealed avoidance of the card with the right eye, and 0.30 cycles per degree in the left eye. A preretinal pale macular lesion, likely an ERM, was noted in the right eye. Indirect ophthalmoscopy during subsequent EUA revealed a dense, white ERM covering the macula and small foci of residual peripheral intraretinal hemorrhages in the right eye (Figure 6, Top left). The left eye showed a normal optic nerve, macula, and retinal vessels. There was no retinal tear or retinal detachment noted in either eye. Hand-held SD OCT of the right eye obtained during EUA revealed a double layer of ERM overlying the fovea with deformation of the retina and apparent vitreomacular traction (Figure 6). SD OCT results of the left eye were within normal limits. A 25-gauge vitrectomy with posterior hyaloid separation and ERM peel was performed on the right eye (Figure 7). A thick ERM was removed surgically, but one month later, the deeper layer of membrane remained with persisting visible foveal deformation evident by SD OCT imaging performed during a follow-up EUA (Figure 8). At that time, a second vitrectomy was performed with removal of the second layer of membrane. SD OCT imaging (Figure 9) was obtained four months later, at the time of electroencephalography diagnostic study under chloral hydrate. The SD OCT system was taken to the electroencephalography suite. The macula had returned to a more normal contour and there was no cystoid edema. At that time, Teller acuity card VA improved to 1.6 cycles per degree with the right eye, 2.4 cycles per degree with the left eye, and 3.1 cycles per degree with both eyes. 


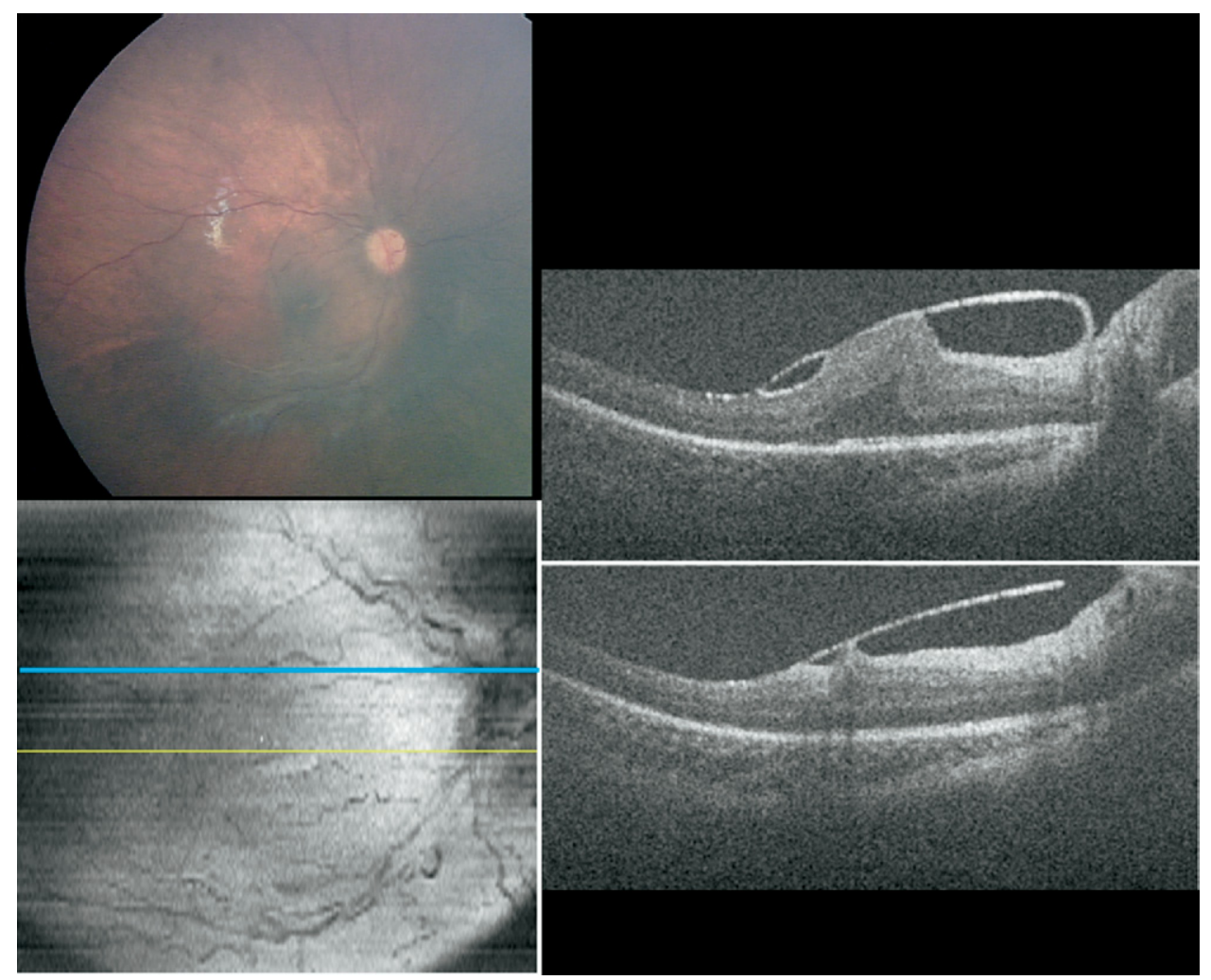

FIGURE 8. (Top left) RetCam color photograph and (Bottom left) enhanced SVP image with a yellow and blue line marking the exact location of the two enhanced B scans top right (yellow line) and bottom right (blue line). (Right) Postoperative SD OCT images of the right eye of the same infant male depicted in Figure 7 show a remaining single layer of ERM. Although there is persistent disruption of the normal foveal architecture, the retina appears to be less thickened.

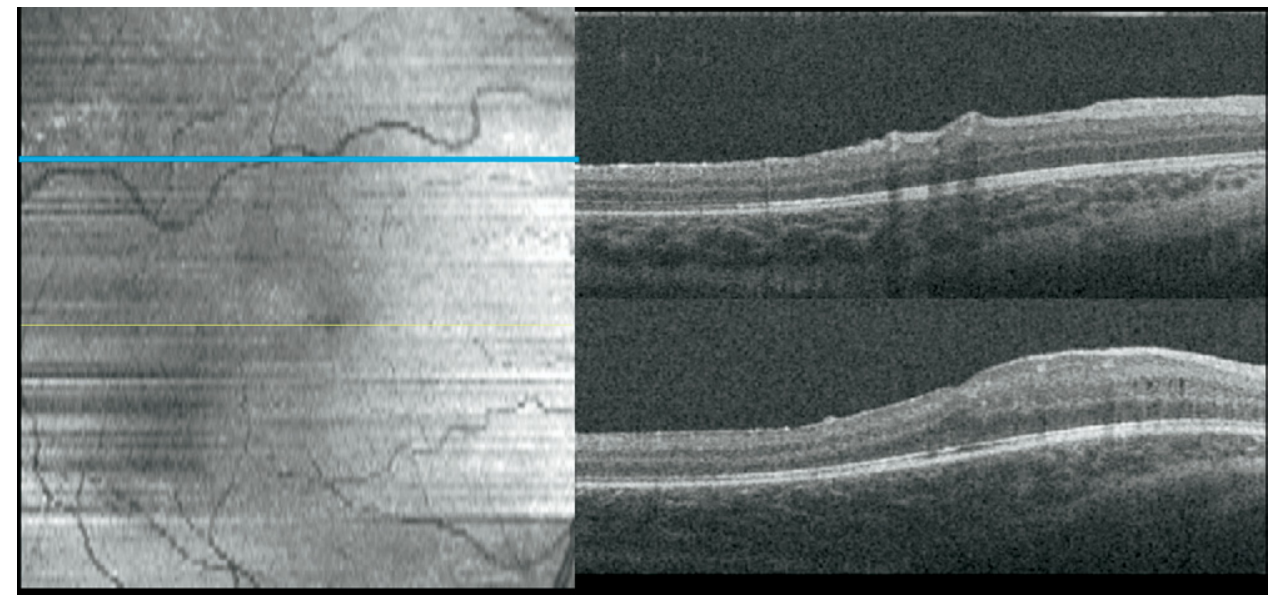

FIGURE 9. (Right) SD OCT images of the right eye of the infant four months after the remaining ERM was removed. The foveal architecture has returned to a more normal configuration. (Left) Enhanced SVP image: the blue and yellow lines correspond to the location of the enhanced B scans, both sweeping from temporal to nasal (blue line is upper right, yellow line is lower right).

\section{DISCUSSION}

OPTICAL COHERENCE TOMOGRAPHY IS A ROUTINE DIAGnostic imaging method for the evaluation of vitreoretinal disease and injury in the adult or cooperative pediatric patient. Anatomic abnormalities not readily apparent on ophthalmoscopy may be imaged with OCT, and the OCT may guide surgical planning or predict visual outcomes. Ultra-high-resolution images of retinal architecture obtained with SD OCT have been shown to detect retinal pathologic features at a higher frequency than other OCT systems. ${ }^{17}$ Despite the potential benefit of OCT imaging of 
the infant retina to evaluate disease processes and injury, until now, it has been difficult to accomplish this with a tabletop chin-rest system.

In this study, imaging of the macula with a hand-held SD OCT probe produced images comparable with those of conventional clinic tabletop SD OCT scanning. Important macular pathologic features were captured during examination of the supine infant (under anesthesia) and adult without special requirement for head or body positioning.

In one infant evaluated for sequelae of SBS, SD OCT aided in the diagnosis of chronic full-thickness macular hole with full-thickness retinal scarring. Findings obtained by SD OCT imaging helped to explain the unilateral vision loss in this infant. Early evaluation with SD OCT imaging may be useful in future patients to identify treatable pathologic features associated with shaken baby trauma.

Macular hole formation has been described in infants with SBS. ${ }^{18}$ One postulated mechanism of full-thickness macular hole formation in this scenario is that the mechanical forces of shaking lead to a structurally weakened retina. Retinal hemorrhage may result in retinal necrosis ${ }^{19}$ and also may cause mechanical pressure on an already weakened retina leading to full-thickness hole formation. ${ }^{18}$ $\mathrm{Ou}$ and associates reported a series of five eyes with macular hole formation as a result of SBS. ${ }^{18}$ In this series, the diagnosis of macular hole was made on initial funduscopic examination results in two patients, during vitrectomy in two patients, and on follow-up funduscopic examination after clearing of vitreous hemorrhage in the final patient. OCT was not used in this study to document or observe the macular holes and may have been useful in guiding surgical planning and predicting visual outcomes. ${ }^{5}$ The presence of macular hole in the setting of SBS is a poor prognostic finding. ${ }^{18}$ Although surgical repair may lead to anatomic success, VA usually remains decreased. ${ }^{18}$

In another infant with intraretinal hemorrhage resulting from shaking trauma but without a history of vitreous or large premacular hemorrhage, a double layer of ERM was visible on SD OCT. The persistence of a second translucent membrane at the time of surgery emphasizes the importance of review and analysis of SD OCT before surgery and the potential benefit of reimaging during surgery to reassess the status of macular pathologic features before closure. With SD OCT, one can appreciate readily the persisting second reflective preretinal layer that was most likely a partially detached internal limiting membrane [ILM] (with or without mild cellular proliferation) with associated foveal deformation at the site of persisting attachment. With SD OCT, one also can appreciate the return to a more normal foveal contour after the second surgery. Although ERM formation as a result of SBS has been described previously, ${ }^{20}$ a double layer of ERM has not been reported in this condition.
Epiretinal membrane has been described as a late manifestation of SBS. ${ }^{20,21}$ One proposed pathophysiologic mechanism of epiretinal formation in this scenario involves acceleration and deceleration forces from shaking of the infant head resulting in trauma to the retinal vessels at sites of firm vitreoretinal attachment such as the interface between the vitreous and ILM in the macular region. The proliferation of glial cells is stimulated by blood in the superficial retinal layers below the ILM. ${ }^{19}$ Breaks in the ILM then permit proliferation of glial cells along the surface of the ILM, forming an ERM that may distort the macular architecture. ${ }^{19,22}$ ERM formation also has been described in Terson syndrome, in which long-standing subhyaloid hemorrhage is postulated to provide a scaffold for glial cell proliferation and ERM formation. ${ }^{23}$ García-Arumí and associates observed a double layer of ERM in six of 11 eyes of patients with Terson syndrome, confirmed by pathologic study: an outer membrane on the posterior vitreous face and an inner ERM overlying the macula, firmly adherent to the ILM. ${ }^{23}$ In this case, a double layer ERM developed without a preceding large subhyaloid hemorrhage, although patchy subhyaloid blood could not be ruled out.

In addition to macular hole and ERM, perimacular retinal folds and traumatic retinoschisis are among the pathologic findings that have been described as a result of pediatric shaking injury. ${ }^{24-28}$ There is debate in the literature as to whether perimacular folds are diagnostic of retinal shaking injury and as to the causal mechanism of these folds. ${ }^{25,29}$ Perimacular folds have been associated with increased morbidity and mortality in children with head injuries caused by abuse. ${ }^{25,30}$ In two eyes, we found perimacular retinal folds, and in one eye, this was associated with posterior vitreous separation and attachment at the site of the white fold. In this case, the SD OCT image did not show a retinal fold, but documented the change in posterior hyaloid attachment at the site of arcuate white reflectivity. Imaging of perimacular arcuate pale reflexes by SD OCT may provide additional insight into whether these lesions are folds or vitreoretinal attachment sites; this may be useful in future evaluation of the perimacular area in eyes of shaken infants.

Traumatic retinoschisis has been described by Greenwald and associates as splitting that occurs within the retinal layers as a result of shaking trauma. ${ }^{27}$ The authors describe, in five babies, macular "intraretinal blood-filled cavities, within which the red blood cells had partially layered out inferiorly." In these eyes, late findings included "extensive gliosis of the retina." Based on the authors' description, it can be argued that these macular lesions may have been subhyaloid hemorrhages, instead of true schisis cavities. The late finding of retinal gliosis likely was ERM resulting from the proliferation of glial cells along the ILM. The conclusion of this study, is that an actual schisis 
cavity can develop in the infant macula as a result of shaking injury, would have been strengthened had SD OCT been available to document the splitting between retinal layers.

Much of the information concerning pathologic retinal findings described in SBS has come from postmortem autopsy pathologic examinations. ${ }^{23-25,31}$ Imaging eyes with SBS with SD OCT would allow the clinician to document retinal abnormalities such as traumatic macular hole, ERM, perimacular folds, and traumatic retinoschisis to provide in vivo information about vitreoretinal abnormalities in these eyes that then may be correlated with the clinical examination results and fundus findings to guide surgical planning and to determine the ultimate prognosis. In this study, we demonstrated imaging of the macula and posterior pole beyond the arcades with the hand-held SD OCT system. Although we did not assess peripheral imaging in this early investigation, we are pursuing this potential application in a study of retinopathy of prematurity.
In conclusion, hand-held SD OCT is a safe, noninvasive, and effective method of obtaining in vivo highresolution information regarding retinal morphologic features, providing a comprehensive macular examination for children during EUA or in conjunction with an examination under sedation. The technique can be used on a sleeping infant, although this is much more challenging than examining the anesthetized child. SD OCT allows the clinician to diagnose and document retinal pathologic features such as a chronic macular hole and ERM many months after the initial trauma and long after retinal hemorrhages have resolved. Documentation of these findings in an infant's eye without any other causative explanation may raise the suspicion of prior shaking injury and may have medicolegal implications. Therefore, SD OCT imaging systems should be considered a useful adjunct to RetCam fundus photography (Clarity Medical Systems Inc, Pleasanton, California, USA) to aid in diagnosis and management in cases of suspected SBS.

THIS STUDY WAS SUPPORTED BY ANGELICA AND EUAN BAIRD, NEW YORK, NEW YORK; A SOFTWARE DONATION FROM BIOPTIGEN Inc; and in part by the North Carolina Biotechnology Center, Research Triangle Park, North Carolina and a Collaborative Grant-2007-CFG-8005 along with Bioptigen Inc, Research Triangle Park, North Carolina. Dr Toth has a patent application pending and receives royalties through Duke University Patent Policy for other technology licensed by Duke University to Alcon Laboratories Inc. Dr Toth has received $\$ 1500$ in consultant fees from Bioptigen Inc. Involved in design of study (C.A.T.); conduct of study (C.A.T., S.F., A.W.S.); provision of patients (C.A.T., L.B.E., D.K.W.); preparation of manuscript (C.A.T., S.F., A.W.S.); and critique and revision of manuscript (C.A.T., S.F., L.B.E., D.K.W., A.W.S.). This study was approved by the Duke University Institutional Review Board. Information was collected and protected in compliance with HIPAA regulations.

\section{REFERENCES}

1. Mirza RG, Johnson MW, Jampol LM. Optical coherence tomography use in evaluation of the vitreoretinal interface: a review. Surv Ophthalmol 2007;52:397-421.

2. Shields CL, Mashayekhi A, Luo CK, Materin MA, Shields JA. Optical coherence tomography in children: analysis of 44 eyes with intraocular tumors and simulating conditions. J Pediatr Ophthalmol Strabismus 2004;41:338-344.

3. Joshi MM, Ciaccia S, Trese MT, Capone A. Posterior hyaloid contracture in pediatric vitreoretinopathies. Retina 2006;26:S38-S41.

4. Patel CK. Optical coherence tomography in the management of acute retinopathy of prematurity. Am J Ophthalmol 2006;141:582-584.

5. Joshi MM, Trese MT, Capone A. Optical coherence tomography findings in stage $4 \mathrm{~A}$ retinopathy of prematurity: a theory for visual variability. Ophthalmology 2006;113:657660.

6. Wojtkowski M, Bajraszewski T, Targowski P, Kowalczyk A. Real-time in vivo imaging by high-speed spectral optical coherence tomography. Opt Lett 2003;28:1745-1747.

7. Yun SH, Boudoux C, Tearney GJ, Bouma BE. High-speed wavelength-swept semiconductor laser with a polygonscanner-based wavelength filter. Opt Lett 2003;28:19811983.

8. Lalwani GA, Punjabi OS, Flynn HW, Knighton RW, Puliafito CA. Documentation of optic nerve pit with macular schisis-like cavity by spectral-domain OCT. Ophthal Surg Lasers Imaging 2007;38:262-264.
9. Punjabi OS, Flynn HW, Legarreta JE, Gregori G, Knighton RW, Puliafito CA. Documentation by spectral-domain OCT of spontaneous closure of idiopathic macular holes. Ophthal Surg Lasers Imaging 2007;38:330-332.

10. Jiao S, Knighton R, Huang X, et al. Simultaneous acquisition of sectional and fundus ophthalmic images with spectral-domain optical coherence tomography. Opt Express 2005;13:444-452.

11. Stopa M, Bower BA, Davies E, Izatt JA, Toth CA. Correlation of pathologic features in spectral-domain OCT imaging with conventional retinal studies. Retina 2008;28:298-308.

12. Farsiu S, Robinson MD, Elad M, Milanfar P. Fast and robust multiframe super resolution. IEEE Trans Image Process 2004; 13:1327-1344.

13. Farsiu S, Chiu SJ, Izatt JA, Toth CA. Duke Optical Coherence Tomography Retinal Analysis Program (DOCTRAP). Provisional Patent Application. Forthcoming.

14. Thévenaz P, Ruttimann UE, Unser M. A pyramid approach to subpixel registration based on intensity. IEEE Trans Image Process 1998; 7:27-41.

15. Farsiu S, Elad M, Milanfar P. Constrained, globally optimal, multi-frame motion estimation. Proc. of IEEE Workshop on Statistical Signal Processing, 2005:1396-1401. Available at http://www.duke.edu/ sf59/motion_ssp.pdf. Accessed: September 16, 2008.

16. Takeda H, Farsiu S, Milanfar P. Kernel regression for image processing and reconstruction. IEEE Trans Image Process 2007;16:349-366.

17. Ko TH, Witkin AJ, Fujimoto JG, et al. Ultra-high-resolution optical coherence tomography of surgically closed macular holes. Arch Ophthalmol 2006;124:827-836. 
18. Ou JI, Moshfeghi DM, Tawansy K, Sears JE. Macular hole in Shaken Baby syndrome. Arch Ophthalmol 2006;124:913-916.

19. Lincoff H, Madjarov B, Lincoff N, et al. Pathogenesis of the vitreous cloud emanating from subretinal hemorrhage. Arch Ophthalmol 2003;121:91-96.

20. Ells AL, Kherani A, Lee D. Epiretinal membrane formation is a late manifestation of Shaken Baby syndrome. J AAPOS 2003; 7:223-225.

21. Harcourt B, Hopkins D. Ophthalmic manifestations of the battered baby syndrome. Br Med J 1971;3:398-401.

22. Burke JM, Smith JM. Retinal proliferation in response to vitreous hemoglobin or iron. Invest Ophthalmol Vis Sci 1981;20:582-592.

23. García-Arumí J, Corcostegui B, Tallada N, Salvador F. Epiretinal membranes in Tersons syndrome: a clinicopathologic study. Retina 1994;14:351-355.

24. Massicotte SJ, Folberg R, Torczynski E, Gilliland MGF, Luckenbach MW. Vitreoretinal traction and perimacular retinal folds in the eyes of deliberately traumatized children. Ophthalmology 1991;98:1124-1127.
25. Marshall DH, Brownstein S, Dorey MW, Addison DJ, Carpenter B. The spectrum of postmortem ocular findings in victims of Shaken Baby syndrome. Can J Ophthalmol 2001; 36:377-384.

26. Lantz PE, Sinal SH, Stanton CA, Weaver RG. Perimacular retinal folds from childhood head trauma. Br Med J 2004; 328:754-756.

27. Greenwald MJ, Weiss A, Oesterle CS, Friendly DS. Traumatic retinoschisis in battered babies. Ophthalmology 1986; 93:618-625.

28. Greenwald MJ, Weiss A, Desterle CS, Friendly DS. Traumatic retinoschisis in battered babies. Ophthalmology 1986; 93:618-625.

29. Chong NV. Perimacular retinal folds and nonaccidental injury: yes, no, or maybe? Eye 2007;21:3-4.

30. Mills M. Fundoscopic lesions associated with mortality in Shaken Baby syndrome. J AAPOS 1998;2:67-71.

31. Elner SG, Elner VM, Arnall M, Albert DM. Ocular and associated systemic findings in suspected child abuse. Arch Ophthalmol 1990;108:1094-1101. 


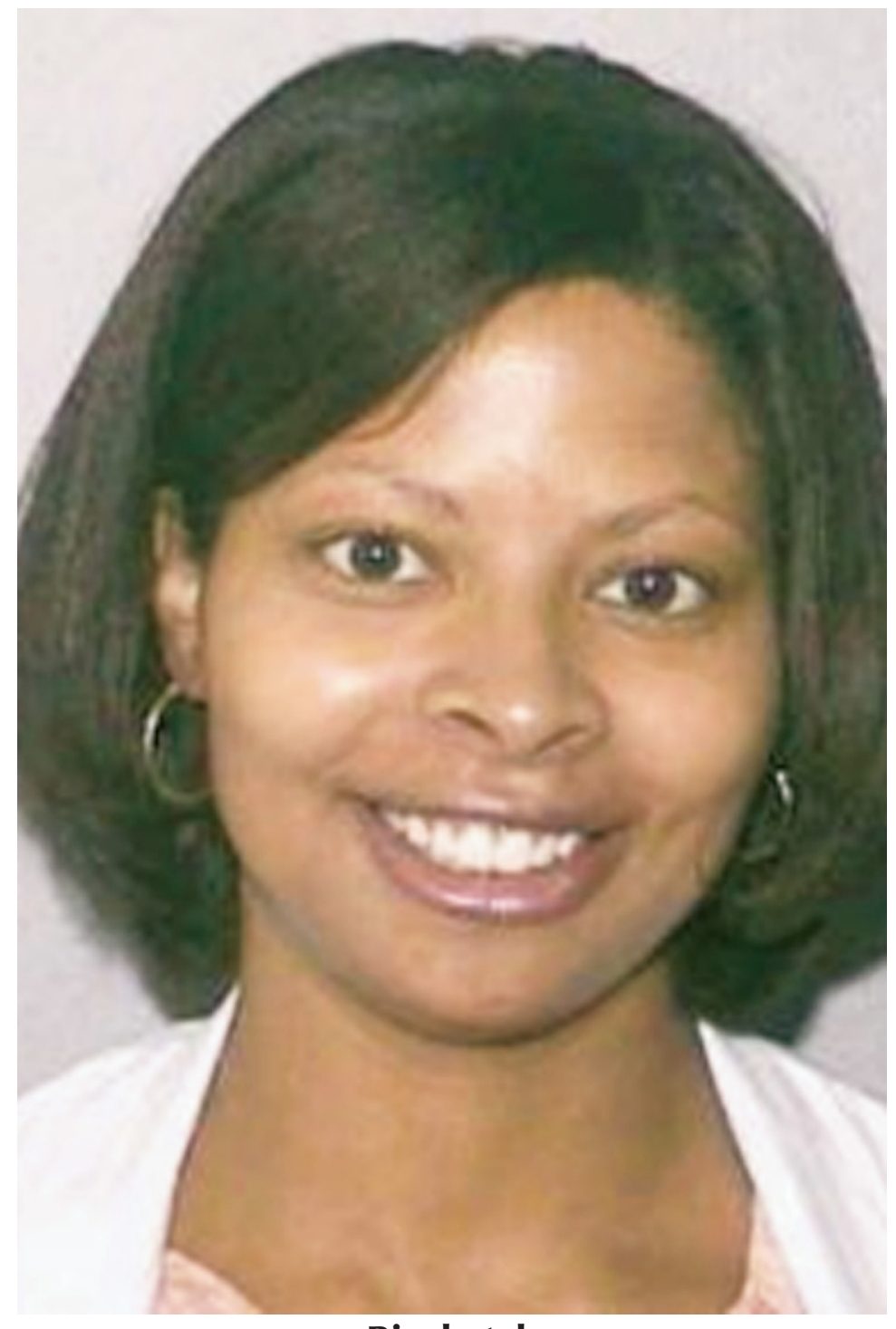

\section{Biosketch}

Dr Adrienne W. Scott recently completed a vitreoretinal fellowship at the Duke University Eye Center in Durham, North Carolina. In August 2008, Dr Scott joined the faculty at the Wilmer Eye Institute at the Johns Hopkins University School of Medicine, Baltimore, Maryland where she is engaged in patient care and clinical research. 


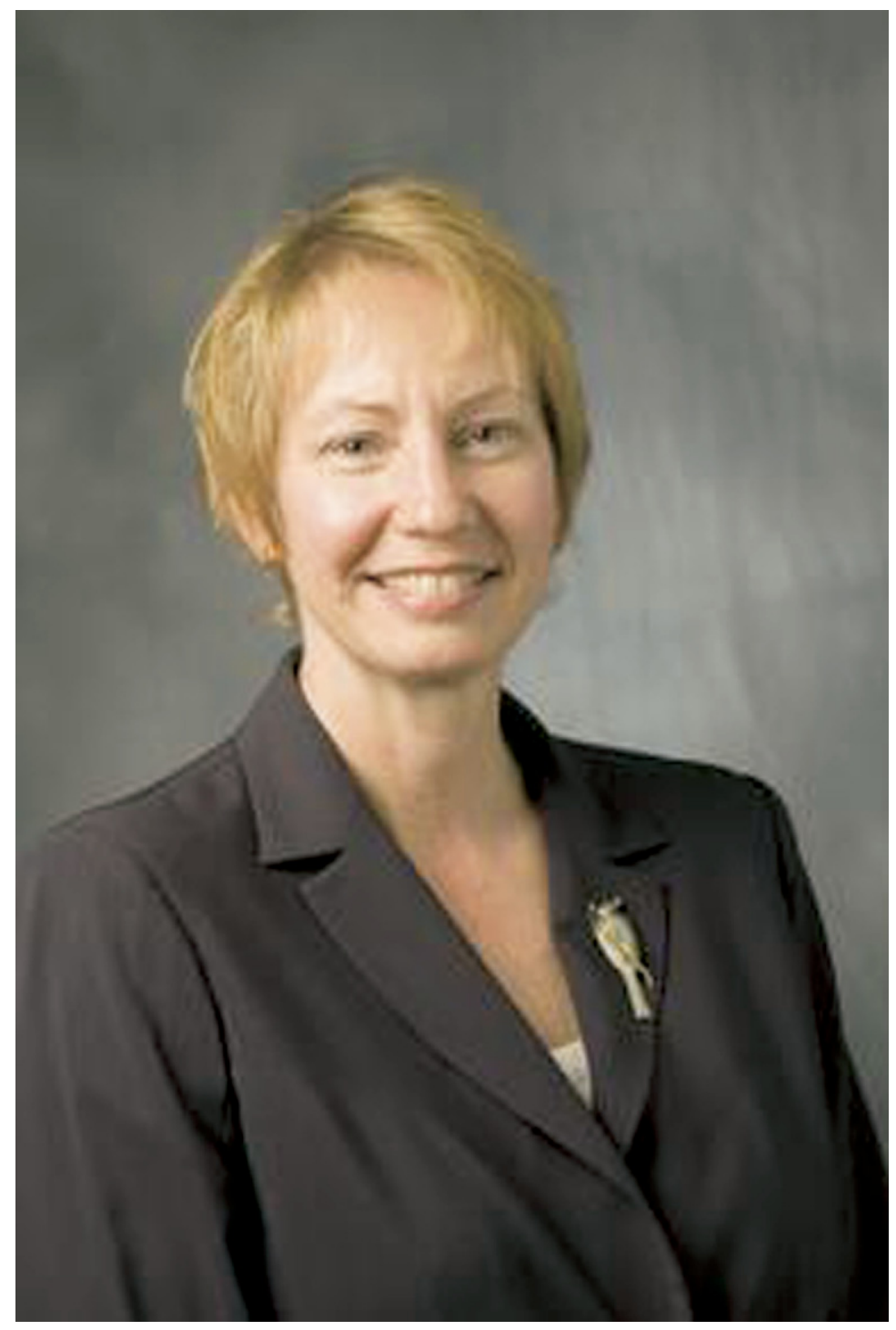

Biosketch

Cynthia A. Toth received her MD from the Medical College of Pennsylvania, Ophthalmology Residency training at Geisinger Medical Center and Fellowship in Vitreoretinal Diseases and Surgery, at University of California, Davis, Davis, California. Dr Toth was Chief of the Retina Service at Wilford Hall USAF Medical Center. Joining Duke University Medical Center Faculty, Durham, North Carolina as a vitreoretinal surgeon in 1993, she is now Professor of Ophthalmology and of Biomedical Engineering. Dr Toth heads the Duke Advanced Research in SDOCT Imaging (DARSI) Laboratory. 